"This is the peer reviewed version of the following article: [International Journal of Mental Health Nursing, 2021, 30, (5), pp. 1070-1079] which has been published in final form at [https://onlinelibrary.wiley.com/doi/10.1111/inm.12859] purposes in accordance with Wiley Terms and Conditions for Self-Archiving." 


\title{
Extending the role of nursing assistants in mental health inpatient settings: a multi-method study
}

Roche, M. A., Glover, S., Luo, X., Joyce, M., \& Rossiter, C. (2021). Extending the Role of Nursing Assistants in Mental Health Inpatient Settings: A Multi-Method Study. International Journal of Mental Health Nursing. https://doi.org/10.1111/inm.12859

\begin{abstract}
With continued growth in the demand for nursing care, many organisations have incorporated nursing assistants into the acute care workforce. However, role descriptions are often generic and do not provide clarity in specialist areas such as mental health inpatient settings. Issues have been noted regarding the role of assistants, delegation, and their integration with the nursing team. This study extended an existing set of care activities and explored the perceptions of nursing assistants and registered nurses regarding these activities. A modified Delphi approach added 14 new care activities for nursing assistants. A follow-up survey found significant differences between nursing assistants and registered nurses regarding utilisation of the activities, delegation, teamwork, and role clarity. Future research must incorporate the perspectives of those with lived experience of mental health issues, and develop an understanding of the interactions between nursing assistant care activities and other factors such as local supports, skillmix, and the practice environment, as these may impact how an organisation can introduce nursing assistants to specialty areas while maintaining consumer and staff safety.
\end{abstract}

\section{Keywords:}

Nursing assistants; mental health; hospital; scope of practice; Delphi 


\section{Introduction}

The need for health workers is expected to continue for the foreseeable future, driven by an increasing and ageing population, greater complexity of care required by hospitalised patients, and increased nursing workloads (Scheffler et al., 2016). Alongside the ageing nursing workforce (WHO, 2016), and substantial pressure to contain costs (Needleman, 2017), this has led to the development and implementation of new models of care, often including the introduction or expansion of unregulated nursing assistants. These workers have various titles internationally, including unlicensed assistive personnel, clinical non-licensed personnel, health care assistants, nurse aides, and assistants in nursing. The generic and inclusive term nursing assistant (NA) will be used in this paper to describe those employed in the health system to deliver a range of ancillary nursing and non-nursing tasks (ACN, 2019). Nursing assistants account for around $25 \%$ of the health workforce in the U.S., U.K., and Australia (AIHW, 2015, WHO, 2016). In Australia, there is no standardisation across states in the education required for this role (ACN, 2019, Blay and Roche, 2020). Qualifications vary but typically include a short training program of up to six months, incorporating both theoretical and practical components. Undergraduate Bachelor of Nursing students may also work in this role during their second or third year of studies (Twigg et al., 2016, NSW Health, 2018).

In Australia, predicted shortages in mental health nurses are greater than in other areas (Ridoutt et al., 2014), providing an even stronger impetus to introduce and expand the role of the unregulated worker. This has seen the introduction of nursing assistants across mental health inpatient settings (Cowan et al., 2015a). Position descriptions and guidelines for these workers are often broad (e.g. NSW Health, 2019) and do not describe activities particular to the specialty, leaving room for role ambiguity and a potential lack of definition in responsibilities (Gabrielsson et al., 2014). Studies in mental health and other areas have identified challenges in delegation, task duplication, delays in care, ambiguous roles, and limited integration with the nursing team (Cleary et al., 2012, Cowan et al., 2015b, Roche et al., 2016, Duffield et al., 2019).

To address some of these challenges, in 2012 a defined set of care activities applicable to specialist mental health inpatient units was developed and implemented across 14 mental health inpatient units (Cowan et al., 2015a). This paper reports on a follow-up study to review, revise, and extend these care activities, and to evaluate their uptake and utility.

\section{Background}

Nursing assistants' roles differ across localities and settings, within boundaries set by legislative, statutory or organisational requirements in the relevant jurisdiction (NSW Health, 2018, NMBA, 2015, National Council of State Boards of Nursing, 2016). Duties typically include a range of direct care activities such as 
assisting with patient meals, bathing, mobilising, and monitoring basic vital signs such as pulse, temperature, and blood glucose, performing their work under the supervision and direction of a registered nurse (RN) (NHS, 2020, NSW Health, 2019). As the practice of hourly 'rounding' becomes more popular in nursing wards, NAs are regularly assigned this task to address fundamental care needs (Ryan et al., 2019), and to report patient care requirements to the RN (NSW Health, 2018, NHS, 2020). These workers are also called upon to undertake close/one-on-one patient surveillance, particularly of patients with challenging behaviour, or who are considered to be at risk of self-harm or violence (Portelli et al., 2018). The lack of standardisation in this role has permitted local flexibility but has also contributed to widely varying implementations and associated challenges in role clarity, delegation, and team integration (Duffield et al., 2019, Bellury et al., 2016, Kalisch, 2011).

Issues with nursing team integration have been identified in other clinical areas. Patient safety and quality care are linked with effective team functioning, which is co-ordinated by three key mechanisms: shared mental models, closed-loop communication and mutual trust (Salas et al., 2005). In general medical surgical and intensive care settings, effective teamwork has been associated with fewer adverse events (Rahn, 2016) and less missed care (Bragadóttir et al., 2017, Chapman et al., 2017). Kalisch and Lee (2013) confirmed this link across settings and identified higher levels of teamwork in mental health units than in intensive care and other units. A perception of stronger teamwork is associated with greater job satisfaction among acute care nursing staff (Kaiser and Westers, 2018), although nursing assistants tend to be less satisfied overall than registered nurses (Kalisch et al., 2010a).

Nursing assistants have reported significantly less team orientation than RNs (Kalisch and Lee, 2013). For example, Bellury and colleagues (2016) identified differences in shared mental models - individual knowledge structures that facilitate collaboration and teamwork (McComb and Simpson, 2014) - between RNs and NAs, with the latter focused on a 'nursing assistant only' team rather than a 'RN-nursing assistant' team. This has implications for communication and delegation, particularly if the NA is to operate effectively under the supervision and direction of a RN (Kærnested and Bragadóttir, 2012). Delegation is a fundamental component of teamwork in this context, and is associated with team functioning, the quality and safety of care, staff retention, and efficient use of nursing and other resources (Kærnested and Bragadóttir, 2012). Improved delegation between RNs and NAs has been linked to fewer falls and greater patient satisfaction in general settings (Wagner, 2018). However, in order to delegate appropriately, RNs benefit from specific education regarding delegation with a sound understanding of the NA's skills and knowledge (White et al., 2011). If these foundations are lacking then there is significant potential to compromise patient safety (Bellury et al., 2016, Kalisch, 2011, Duffield et al., 2019). It is reasonable to suggest that ineffective teamwork and delegation are central factors in the negative outcomes often seen 
when NAs have been introduced to acute care environments (Aiken et al., 2016, Twigg et al., 2016, Duffield et al., 2020).

Complicating teamwork and delegation are questions of role clarity. A systematic review of NAs' tasks across many inpatient settings (Blay and Roche, 2020) found evidence that some activities were beyond NAs' training and skill level, and proposed that this could be a consequence of task duplication (Roche et al., 2017) or of NAs' belief that their role was similar to that of a registered nurse (Gerace et al., 2018). In mental health settings, Cleary and colleagues (2012) explored the role of NAs and found that these workers were focused on performing tasks consistent with practice in other clinical settings, such as vital signs and activities of daily living. They also found issues of support and role ambiguity, and suggested that mental health-specific knowledge, such as understanding psychiatric medication and behavioural indicators, could provide greater clarity and team integration, thereby improving the utilisation of these personnel (Cleary et al., 2012). Some research has suggested that NAs in mental health settings can prioritise contact with consumers and thereby establish strong relationships (Gabrielsson et al., 2014, Gabrielsson et al., 2016). However, this is not universal, as in other clinical areas the introduction of additional staff in the form of NAs did not enhance satisfaction with emotional care provided by nurses (Duffield et al., 2018, Duffield et al., 2019).

Registered nurses in mental health settings have described their expectations that NAs take on a broader set of activities (Cleary et al., 2012, Gabrielsson et al., 2016). Driven by these expectations and the potential to improve role clarity, Cowan and colleagues (2015a) developed a defined set of activities for NAs in inpatient mental health using a modified Delphi methodology. In that study, consensus was reached not only for activities consistent with general activities such as taking and recording vital signs, but also for more mental health-specific activities such as promoting and maintaining a safe environment, group programs, and participating in aggression response teams. Understanding the implementation of such context-specific activities is vital in order to assess their impact on role clarity, teamwork, and delegation, and to provide a framework on which to build implementation training and ongoing support (Cowan et al., 2015a).

\section{Aim}

There were two aims for this study. The first was to extend the existing set of care activities for nursing assistants in mental health inpatient units. The second was to explore the perceptions of nursing assistants and registered nurses regarding these activities, specifically, to:

- Assess the utilisation of the extended care activities

- Describe delegation and the clarity of the nursing assistant role

○ Measure nursing teamwork 


\section{Method}

This two-phase study was undertaken in 14 inpatient mental health units in one metropolitan health service, in New South Wales (NSW), Australia. Phase 1 comprised a Delphi study to meet the first aim, followed by Phase 2, a survey of nurses and nursing assistants to address the second aim (Error! Reference source not found.).

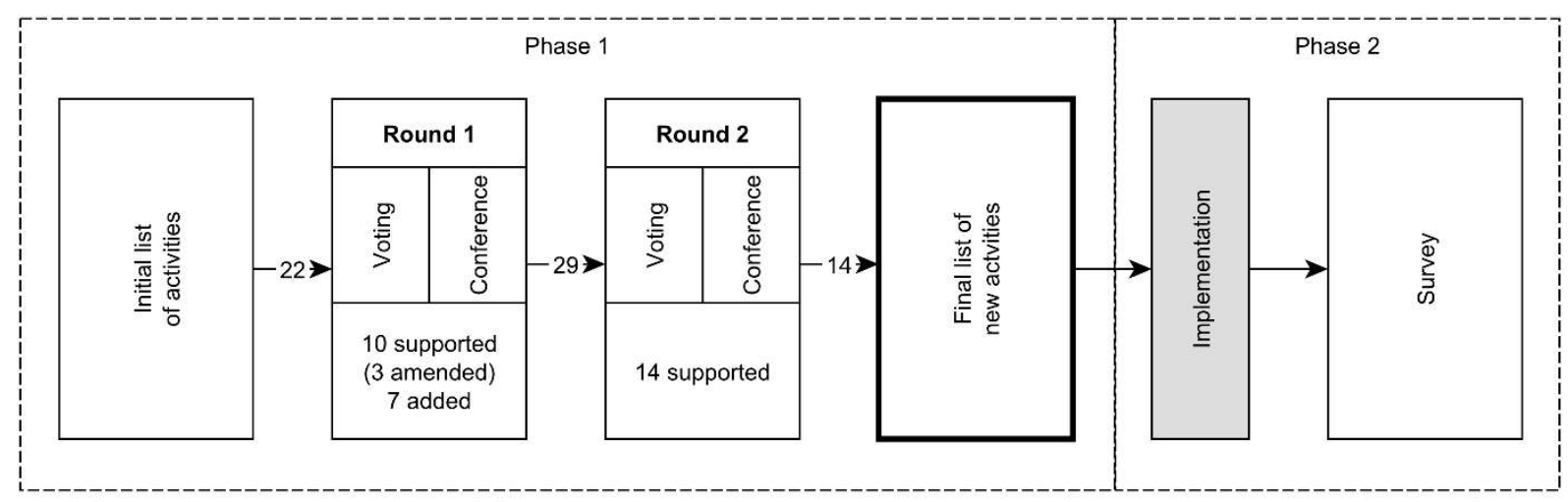

Figure 1 Study Design

\section{Phase 1}

Phase 1, the development of a set of extended care activities, occurred between April and July 2016 using a modified Delphi approach that combined voting rounds with consensus conferencing (Kizawa et al., 2012, Hasson et al., 2000) among 10 stakeholders in accordance with the method used in the initial development study (Cowan et al., 2015a). This phase of the project was assessed by the organisation's Human Research Ethics Committee as 'an activity not requiring HREC review'.

\section{Panel selection}

Purposive sampling was used to invite experts to the process, individuals identified by the researchers to have experience in working with, teaching, and supervising these staff. This process led to a final panel of ten: nurse managers (2), educators internal and external to the organisation (3), clinical nurses (3), and nursing assistants (2). Ten was considered appropriate as large numbers have been linked to coordination problems, while panels of less than six may lead to validity and reliability issues (Black et al., 1999). To ensure quality, each member's expert status was confirmed through assessing the degree of their involvement with NAs, and/or their length of experience in a relevant position (Paré et al., 2013).

\section{Procedure}

This modified Delphi approach typically requires between two and six rounds of voting and conference to reach consensus (Kizawa et al., 2012, Cowan et al., 2015a), with consensus in this study indicated by $70 \%$ 
agreement on an item. A working group of senior managers and clinical nurses developed a list of 22 care activities that extended the current practice list for nursing assistants of 25 activities developed by Cowan et al. (2015a). This set of activities established a common perspective and provided the group with a framework for discussion and the identification of potential additional activities. This is referred to as a 'straw model', which assists participants in the Delphi process to collaborate towards consensus (Adler and Ziglio, 1996). Panellists were provided with this list and asked to vote on whether they believed the items were appropriate for NAs ('Yes', 'Yes with training', or 'No'), with the first two responses considered affirmative and the last negative. The inclusion of 'with training' indicated that they believed the activity was suitable if the assistant received specific training. Stakeholders reviewed voting at the conference and discussed each item individually, with opportunity to amend activity descriptors, add items and revise voting (Vázquez-Ramos et al., 2007). In round 2, panel members received the revised list of activities, including scores from the previous round, followed by a second conference. Once consensus of $70 \%$ or more was reached, there was no requirement for further rounds (Kizawa et al., 2012, Vázquez-Ramos et al., 2007), leading to two complete rounds in this study.

\section{Implementation}

The activities identified in Phase 1 were incorporated into all nursing orientation activities and position descriptions for NAs. The central NA skill development and education program, held twice annually, reinforced the activities. A standardised presentation and detailed flyer that listed all activities with colourcoding were developed and posted in easily accessible locations on all units. Briefings were held on all 14 units to describe and discuss the activities with RNs and other staff, with opportunity for clarification. All implementation activities were complete before Phase 2 commenced.

\section{Phase 2}

Phase 2 was undertaken from June to December 2018 using a descriptive survey design. Both nursing assistants and registered nurses were anonymously surveyed using Qualtrics ${ }^{\mathrm{TM}}$ online survey software. This phase of the study received HREC approval (LNR/18/HAWKE/6).

\section{Recruitment}

Flyers notifying nurses of the study were distributed to all 14 inpatient units, a notice placed in the service newsletter, and on the service Facebook page. Potential participants were sent a link to the online survey via email, with reminders made at team meetings. A precise figure for potential participants was not available but this was estimated at 400 . Forty-five valid responses were received, an approximate response rate of $11.3 \%$. 


\section{Survey Instruments}

The survey included the Nursing Teamwork Scale (NTS) (Kalisch et al., 2010b), five items adapted from the Preparedness to Delegate Scale (Kærnested and Bragadóttir, 2012) and six from the Role Ambiguity scale (Rizzo et al., 1970). The delegation and ambiguity items were chosen to provide a concise assessment of those factors while limiting respondent burden. Wording was modified from the original in order to address the different perspectives of RNs and nursing assistants. For example, a delegation item for RNs read "When delegating, do you make clear who is to do the task?" while for NAs it read "When delegating, do RNs make it clear who is to do the task?" Regarding utilisation, participants were asked whether they were aware of the care activities ('yes' or 'no'), and whether the care activities were used as a guide to practice (responses from 'never' to 'always'). The NTS is a 33-item Likert-type questionnaire with established validity in inpatient nursing settings (Kalisch and Lee, 2013), informed by a 5-component model of teamwork (Salas et al., 2005). It has good psychometric properties and measures five domains: backup (team members' willingness to assist one other), shared mental model (understanding of shared roles and responsibilities), team leadership (monitoring and distribution of workload), trust (communication of ideas and seeking feedback), and team orientation (working together to address weaknesses). It also provides an overall teamwork score (Kalisch et al., 2010b).

\section{Analysis}

Data were analysed descriptively and presented as frequency and percent or as means where appropriate. Subscales for the NTS were calculated as means (Kalisch and Lee, 2013). Items from the Preparedness to Delegate Scale and Role Ambiguity were treated as individual questions. Missing data were ignored listwise. Contrasts were drawn between nursing assistants and registered nurses where feasible, but statistical tests were not undertaken because of the small sample and unequal group sizes.

\section{Results}

Results are presented by phase and in accordance with the aims.

\section{Phase 1}

Round one voting indicated support for five of the 22 initial activities pertaining to documentation, observation, and escort duties (Table 1). The conference confirmed support for these activities and added support for a further five. Three tasks were amended for clarity or to specify a condition on the activity, for example patient escort off-campus to be undertaken only with the approval of the nurse in-charge. An additional seven activities were proposed, leading to an expanded list of 29 activities. All items were carried forward to the next round to allow the panel to confirm or refute their earlier consensus. Second round voting found consensus for seven of the 10 supported in round one, plus all seven of the new items. Notably, consensus was not reached for three of the items that had been supported in the first round: 
completing a pain scale, admission documentation, and carrying the aggression response pager. The final list of activities comprised 14 items across a wide span such as mental health nursing observations, escorting patients, documentation, assessment tasks, and administrative duties. A communication strategy at the unit level was undertaken to inform and educate RNs and NAs regarding the activities, and amendments made to position descriptions where required.

Table 1 Summary of Voting and Conference Rounds - Phase 1

\begin{tabular}{|c|c|c|c|c|c|c|c|}
\hline & Round 1 & Vote & Conf. & Round 2 & Vote & Conf. & Final \\
\hline \multirow{6}{*}{ 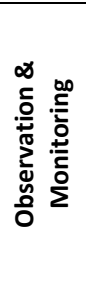 } & Alcohol withdrawal assessment & $43 \%$ & $43 \%$ & Alcohol withdrawal assessment & $13 \%$ & $13 \%$ & \\
\hline & Neurovascular assessment & $29 \%$ & $29 \%$ & Neurovascular assessment & $13 \%$ & $13 \%$ & \\
\hline & $\begin{array}{l}\text { Electrocardiogram (conduct, not } \\
\text { interpret) }\end{array}$ & $57 \%$ & $70 \%$ & $\begin{array}{l}\text { Electrocardiogram (conduct, not } \\
\text { interpret) }\end{array}$ & $88 \%$ & $88 \%$ & $\checkmark$ \\
\hline & Peak expiratory flow rate & $43 \%$ & $70 \%$ & Peak expiratory flow rate & $88 \%$ & $88 \%$ & $\checkmark$ \\
\hline & Pain score & $71 \%$ & $71 \%$ & Pain score & $50 \%$ & $50 \%$ & \\
\hline & & & & $\begin{array}{l}\text { All levels of mental health } \\
\text { nursing observations }{ }^{a}\end{array}$ & $88 \%$ & $88 \%$ & $\checkmark$ \\
\hline \multirow{4}{*}{ 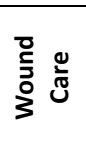 } & Complex dressing & $29 \%$ & $29 \%$ & Complex dressing & $13 \%$ & $13 \%$ & \\
\hline & Wound debridement & $29 \%$ & $29 \%$ & Wound debridement & $0 \%$ & $0 \%$ & \\
\hline & Wound packing & $14 \%$ & $14 \%$ & Wound packing & $25 \%$ & $25 \%$ & \\
\hline & Removal of sutures/clips & $29 \%$ & $29 \%$ & Removal of sutures/clips & $13 \%$ & $13 \%$ & \\
\hline \multirow{3}{*}{ 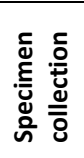 } & Remove sputum via trap & $57 \%$ & $57 \%$ & Remove sputum via trap & $13 \%$ & $13 \%$ & \\
\hline & $\begin{array}{l}\text { Remove urine via indwelling } \\
\text { catheter }\end{array}$ & $57 \%$ & $57 \%$ & $\begin{array}{l}\text { Remove urine via indwelling } \\
\text { catheter }\end{array}$ & $13 \%$ & $13 \%$ & \\
\hline & Wound specimen collection & $57 \%$ & $57 \%$ & Wound specimen collection & $38 \%$ & $38 \%$ & \\
\hline \multirow{8}{*}{ 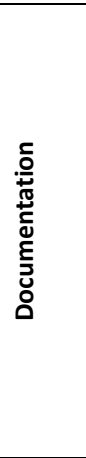 } & $\begin{array}{l}\text { Admission (nursing bio-psycho- } \\
\text { social history) }\end{array}$ & $29 \%$ & $70 \%$ & $\begin{array}{l}\text { Admission (nursing bio-psycho- } \\
\text { social history) }\end{array}$ & $50 \%$ & $50 \%$ & \\
\hline & Medication chart \& discharge & $43 \%$ & $43 \%$ & Medication chart \& discharge & $13 \%$ & $13 \%$ & \\
\hline & $\begin{array}{l}\text { Waterlow pressure risk } \\
\text { assessment scale }\end{array}$ & $71 \%$ & $71 \%$ & $\begin{array}{l}\text { Waterlow pressure risk } \\
\text { assessment scale }\end{array}$ & $75 \%$ & $75 \%$ & $\checkmark$ \\
\hline & Consumer daily nursing report & $57 \%$ & $70 \%$ & $\begin{array}{l}\text { Documentation and handover of } \\
\text { own observations and actions }^{\text {b }}\end{array}$ & $88 \%$ & $88 \%$ & $\checkmark$ \\
\hline & $\begin{array}{l}\text { Entering incident management } \\
\text { system report }\end{array}$ & $86 \%$ & $86 \%$ & $\begin{array}{l}\text { Entering incident management } \\
\text { system report }\end{array}$ & $88 \%$ & $88 \%$ & $\checkmark$ \\
\hline & & & & Ontario fall risk screen ${ }^{\mathrm{a}}$ & $100 \%$ & $100 \%$ & $\checkmark$ \\
\hline & & & & Consumer leave $\&$ return ${ }^{a}$ & $88 \%$ & $88 \%$ & $\checkmark$ \\
\hline & & & & $\begin{array}{l}\text { Consumer belongings and } \\
\text { valuables }^{\mathrm{a}}\end{array}$ & $100 \%$ & $100 \%$ & $\checkmark$ \\
\hline \multirow{2}{*}{ 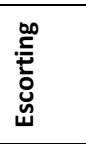 } & Forensic consumers on campus & $71 \%$ & $71 \%$ & $\begin{array}{l}\text { Forensic consumers on campus } \\
\text { with a Registered Nurse }\end{array}$ & $100 \%$ & $100 \%$ & $\checkmark$ \\
\hline & All consumers off campus & $86 \%$ & $86 \%$ & $\begin{array}{l}\text { All consumers off campus as } \\
\text { approved by Nurse-in-Charge }\end{array}$ & $88 \%$ & $88 \%$ & $\checkmark$ \\
\hline \multirow{4}{*}{ 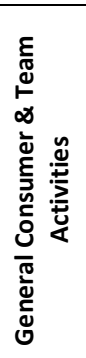 } & Medication administration & $14 \%$ & $14 \%$ & Medication administration & $0 \%$ & $0 \%$ & \\
\hline & & & & $\begin{array}{l}\text { Consumer orientation to the } \\
\text { ward }^{\text {a }}\end{array}$ & $100 \%$ & $100 \%$ & $\checkmark$ \\
\hline & & & & $\begin{array}{l}\text { Issuing and returning consumer } \\
\text { belongings }{ }^{\mathrm{a}}\end{array}$ & $88 \%$ & $88 \%$ & $\checkmark$ \\
\hline & & & & $\begin{array}{l}\text { Precept less experienced and } \\
\text { new AINs }{ }^{\mathrm{a}}\end{array}$ & $88 \%$ & $88 \%$ & $\checkmark$ \\
\hline \multirow{2}{*}{ 高 } & $\begin{array}{l}\text { Carrying the aggression response } \\
\text { team pager }\end{array}$ & $57 \%$ & $70 \%$ & $\begin{array}{l}\text { Carrying the aggression response } \\
\text { team pager }\end{array}$ & $25 \%$ & $25 \%$ & \\
\hline & Checking emergency trolley & $57 \%$ & $57 \%$ & Checking emergency trolley & $13 \%$ & $13 \%$ & \\
\hline
\end{tabular}

a Items added following Round 1

$b$ Items amended following Round 1 (amendments italicised) 


\section{Phase 2}

The 45 surveys returned were largely complete. Respondents were young relative to the population of nurses working in mental health in Australia (32 vs 47 years) and with an average of 5 years' experience (Table 2). Nursing assistants were older with a larger proportion employed part time.

Table 2 Respondent Characteristics - Phase 2

\begin{tabular}{|c|c|c|c|}
\hline & $\begin{array}{c}\text { RNs } \\
(n=36)\end{array}$ & $\begin{array}{l}\text { NAs } \\
(n=9)\end{array}$ & $\begin{array}{l}\text { Overall } \\
(n=45)\end{array}$ \\
\hline & Mean (SD) & Mean (SD) & Mean (SD) \\
\hline Age & $30.5(7.83)$ & $36.3(14.26)$ & 32 (9.99) \\
\hline Experience in Mental Health (years) & $5.2(3.57)$ & $4.1(1.57)$ & $5(3.23)$ \\
\hline Employment status & N (\%) & $\mathbf{N}(\%)$ & $\mathrm{N}(\%)$ \\
\hline Full time & $24(68.6 \%)$ & $2(22.2 \%)$ & $26(59.1 \%)$ \\
\hline Part time & $11(31.4 \%)$ & $6(66.7 \%)$ & $17(38.6 \%)$ \\
\hline Casual & $0(0 \%)$ & $1(11.1 \%)$ & $1(2.3 \%)$ \\
\hline
\end{tabular}

Note: Missing data varies by item

Most participants overall (80\%; Table 3) indicated they were aware of the activities, with a similar proportion (71\%) stating that the activities were used as a guide to practice. However, there was a clear difference between RNs and NAs, particularly for the latter question, with two-thirds of NAs noting they were never or rarely used. More than a quarter of RNs stated the activities were often or always used, while no NAs reported that frequency of use. Similarly, there were clear differences in RNs' and NAs' views on delegation, with over $80 \%$ of RNs stating it was often or always clear who is to undertake a task and nearly $90 \%$ of NAs saying that this was a rare or sometimes occurrence. For NAs, a similar picture was seen for goals and directions, but greater clarity was evident for responsibilities, expectations, explanations, and authority. 
Table 3 Utilisation, Delegation, and Role Ambiguity - Phase 2

\begin{tabular}{|c|c|c|c|}
\hline & $\begin{array}{c}\text { RNs } \\
(n=36) \\
N(\%) \\
\end{array}$ & $\begin{array}{l}\text { NAs } \\
(n=9) \\
N(\%) \\
\end{array}$ & $\begin{array}{c}\text { Overall } \\
\text { (n=45) } \\
N(\%)\end{array}$ \\
\hline Are you aware of the extended care activities? & $30(83.3 \%)$ & $6(66.7 \%)$ & $36(80.0 \%)$ \\
\hline \multicolumn{4}{|l|}{ Are the extended care activities used as a guide to practice? } \\
\hline Always & $1(2.8 \%)$ & $0(0.0 \%)$ & $1(2.2 \%)$ \\
\hline Often & $9(25.0 \%)$ & $0(0.0 \%)$ & $9(20.0 \%)$ \\
\hline Sometimes & $19(52.8 \%)$ & $3(33.3 \%)$ & $22(48.9 \%)$ \\
\hline Rarely & $5(13.9 \%)$ & $1(11.1 \%)$ & $6(13.3 \%)$ \\
\hline Never & $2(5.6 \%)$ & $5(55.6 \%)$ & $7(15.6 \%)$ \\
\hline \multicolumn{4}{|l|}{ When delegating, is it clear... } \\
\hline ...who is to do the task? & $30(83.3 \%)$ & $1(11.1 \%)$ & $31(68.9 \%)$ \\
\hline ...when to do the task? & $35(97.3 \%)$ & $1(11.1 \%)$ & $36(80.0 \%)$ \\
\hline ...where to do the task? & $29(80.6 \%)$ & $1(11.1 \%)$ & $30(66.7 \%)$ \\
\hline ...why to do the task? & $17(47.2 \%)$ & $1(11.1 \%)$ & $18(40.0 \%)$ \\
\hline ...how to do the task? & $23(63.8 \%)$ & $1(11.1 \%)$ & $24(53.4 \%)$ \\
\hline \multicolumn{4}{|l|}{ Role Ambiguity } \\
\hline Clear, planned goals and objectives exist for the NA position & $21(58.4 \%)$ & $2(22.2 \%)$ & $23(51.1 \%)$ \\
\hline NAs do not receive incompatible requests from two or more people ${ }^{a}$ & $11(30.6 \%)$ & $0(0.0 \%)$ & $11(24.4 \%)$ \\
\hline I know what the responsibilities of NAs are & $35(97.2 \%)$ & $9(100.0 \%)$ & $44(97.8 \%)$ \\
\hline I know exactly what is expected of NAs & $32(88.9 \%)$ & $8(88.9 \%)$ & 40 (88.8\%) \\
\hline NAs receive clear explanations of what work has to be done & $20(55.6 \%)$ & $8(88.9 \%)$ & $28(62.2 \%)$ \\
\hline I feel certain about how much authority NAs have & $25(69.5 \%)$ & $5(55.5 \%)$ & $30(66.7 \%)$ \\
\hline
\end{tabular}

a This item was reverse coded for analysis; original wording: 'NAs receive incompatible requests from two or more people'

Note: Missing data varies by item

Contrasts were also apparent between NAs and RNs regarding teamwork (Table 4). Statistical tests were not undertaken but higher scores were noted for NAs in all domains, particularly regarding trust, team orientation, and backup behaviour.

Table 4 Nursing Teamwork Scale - Phase 2

\begin{tabular}{lccc}
\hline & RNs & NAs & Overall \\
\hline Backup & $3.6(0.46)$ & $3.9(0.15)$ & $\mathbf{3 . 7}(\mathbf{0 . 4 3 )}$ \\
Shared Mental Model & $3.7(0.40)$ & $3.8(0.30)$ & $\mathbf{3 . 7}(\mathbf{0 . 3 8 )}$ \\
Leadership & $3.7(0.59)$ & $3.9(0.48)$ & $\mathbf{3 . 8 ( 0 . 5 7 )}$ \\
Team Orientation & $3.5(0.52)$ & $3.8(0.18)$ & $\mathbf{3 . 6 ( 0 . 4 8 )}$ \\
Trust & $3.5(0.73)$ & $4.1(0.34)$ & $\mathbf{3 . 6 ( 0 . 7 1 )}$ \\
\hline Teamwork Overall & $\mathbf{3 . 6 ( 0 . 4 7 )}$ & $\mathbf{3 . 9 ( 0 . 1 5 )}$ & $\mathbf{3 . 7 ( 0 . 4 4 )}$ \\
\hline
\end{tabular}

\section{Discussion}

The increased use of NAs in mental health challenges managers to effectively integrate these staff into the workplace and to confirm they are operating effectively and competently within the limits of their skill level 
and education. Generic guidelines provide limited specific direction and Phase 1 of this study identified a range of activities specific to the mental health context, while Phase 2 found issues with utilisation and disparity between the perceptions of RNs and NAs regarding delegation, role clarity and teamwork. Together, the findings provide a foundation and a process for the development of specialty-specific activities for NAs, while highlighting some of the challenges that need further consideration, particularly the need to ensure strong local support for both NAs and RNs, and to establish and sustain a collective understanding of both the model of care and of the limitations on practice.

The preparation and implementation of NA positions has developed outside a regulatory framework leading to considerable variation in education and task span (Blay and Roche, 2020). This has been noted in independent reviews that have recommended NAs receive more robust education and are accredited or regulated similar to other health professions (Schwartz, 2019, ACN, 2019). The modified Delphi component of this study may contribute to these processes through the establishment of a list of context-specific activities that can be communicated clearly, and incorporated into orientation, education, local support processes, and position descriptions. When such a set of activities is developed with consideration of other local factors such as the unit's skillmix and practice environment, they can also guide RNs regarding which tasks can be safely delegated to NAs.

However, despite the potential benefits of a defined set of activities and apparent clarity regarding responsibilities and expectations, this study found substantial differences in the perceived utilisation of activities between RNs and NAs, and that all NAs received incompatible requests from two or more people. These findings may be a manifestation of the centrality of the activities to NAs or of their opportunity for reinforcement, as the NAs discussed and developed a stronger understanding of the activities during their skill development sessions. This suggests that RNs would benefit from a similar level of support and reinforcement. Divergent views between RNs and NAs regarding the delegation of tasks may reflect previous work that found NAs consider teamwork in a NA-centric manner rather than a comprehensive nursing team approach (Bellury et al., 2016). Indeed, while the overall teamwork score was higher than that reported by Kaiser and Westers (2018), particularly regarding team orientation, it is not clear if the focus of that orientation is NA-centric or comprehensive. The divergence is particularly concerning due to the wide discrepancy in views and the consequent potential for misunderstanding and either duplication or missed care as noted in other clinical areas (Roche et al., 2016, Roche et al., 2017). Challenges in delegation and integration of NAs have been identified in Australia across general and mental health nursing settings (Cleary et al., 2012, Cowan et al., 2015b, Duffield et al., 2019), and linked to increased workloads for RNs (Duffield et al., 2020). While the impact of these challenges has not been established in mental health, in light of the negative consumer and nurse outcomes found in non-mental health contexts (Aiken et al., 2016, Twigg et al., 2016, Duffield et al., 2020), there is the potential for similar consequences. A shared 
understanding of the team and of the model of care is central to patient safety and workplace efficiency, and future developments must address this issue.

A final aspect for consideration in this study is the overall level of experience in mental health of the RN sample. While this must be viewed in light of the small sample size that may not be representative of the service or nurse working in mental health broadly, it remains important to recognise that NAs need close support from RNs with appropriate experience and qualifications to model and facilitate best practice (Gerace et al., 2018).

\section{Limitations}

There are several limitations to this study and findings should be viewed with these in mind. In the modified Delphi study, the initial 'straw model' provided a practical starting point but may also be seen as a boundary that limited the number of potential activities. While expansion of this model from the literature would have been desirable, no material was located that augmented the model. It is also important to note that the Delphi study sought to extend a set of activities for NAs working in mental health inpatient units and made no assessment of ward skillmix, acuity, or workload, important factors that should be assessed before applying these findings. A further consideration is the nature of the stakeholders, who were all health professionals from one mental health service. Expansion of the Delphi panel to include people with lived experience of mental health issues and their support people, and across multiple settings and services, would be an essential addition to future research. The survey component was impacted by the small response rate and overall sample size and was not representative of nurses working in mental health in Australia, limiting generalisability. Finally, the survey did not capture mental health qualifications, and was partly comprised of items derived from instruments rather than the complete tools. Analytical options were therefore restricted.

\section{Conclusion}

The introduction of nursing assistants into any clinical area must incorporate the body of research that has identified the performance of tasks outside NAs' level of skill and education (Blay and Roche, 2020, Duffield et al., 2018, Roche et al., 2017, McNally and Blay, 2018), the hidden RN workload in delegation and supervision (Duffield et al., 2020), the risks associated with replacement of RNs with NAs (Aiken et al., 2016), and the potential for negative patient outcomes even when NAs are added above baseline staffing (Twigg et al., 2016). Nonetheless, NAs have a role in mental health care and there remains an ongoing imperative to understand the activities they undertake, how they integrate with the nursing team, and how this change in skillmix can be most effectively managed to optimise patient safety and job satisfaction. Potentially, the implementation of detailed, context-specific activities alongside high-quality support processes could provide mental health services with the opportunity to incorporate NAs without the 
negative outcomes observed in other clinical areas. To work towards this desirable outcome, future work will need to incorporate the perspectives of consumers, carers, and health service managers, and work towards identifiable outcomes that can be attributed to the work of NAs in mental health.

\section{Relevance for Clinical Practice}

There is limited context-specific guidance for nursing assistants in mental health. The process used to derive the defined activities described in this study may be applied in many services. The considerable disparity between the views of nursing assistants and registered nurses identified in this study suggests the needs for whole-of-team education and expanded support mechanisms to ensure nursing assistants are effectively integrated with the team. Further, while the implications of leaner skillmix in mental health inpatient settings are not well explored, given the large body of evidence in general nursing that has found negative outcomes from such a change, mental health services should introduce NAs with caution and monitor closely. 


\section{References}

ACN (2019). Regulation of the Unregulated Health Care Workforce across the health care system - A White Paper by ACN 2019. Canberra: Australian College of Nursing

AIHW (2015). Health Workforce. Canberra: Australian Institute of Health and Welfare.

Adler, M., \& Ziglio, E. (1996). Gazing into the oracle: The Delphi method and its application to social policy and public health. Jessica Kingsley Publishers.

Aiken, L. H., Sloane, D. M., Griffiths, P., et al. (2016). Nursing skill mix in European hospitals: cross-sectional study of the association with mortality, patient ratings, and quality of care. BMJ Quality \& Safety, 26, 559-568.

Bellury, L., Hodges, H., Camp, A. \& Aduddell, K. (2016). Teamwork in Acute Care: Perceptions of Essential but Unheard Assistive Personnel and the Counterpoint of Perceptions of Registered Nurses. Research in Nursing \& Health, 39, 337-346.

Black, N., Murphy, M., Lamping, D., et al. (1999). Consensus Development Methods: A Review of Best Practice in Creating Clinical Guidelines. Journal of Health Services Research \& Policy, 4, 236-248.

Blay, N. \& Roche, M. A. (2020). A systematic review of activities undertaken by the unregulated Nursing Assistant. Journal of Advanced Nursing, 76, 1538-1551.

Bragadóttir, H., Kalisch, B. J. \& Tryggvadóttir, G. B. (2017). Correlates and predictors of missed nursing care in hospitals. Journal of Clinical Nursing, 26, 1524-1534.

Chapman, R., Rahman, A., Courtney, M. \& Chalmers, C. (2017). Impact of teamwork on missed care in four Australian hospitals. Journal of Clinical Nursing, 26, 170-181.

Cleary, M., Horsfall, J., O'Hara-Aarons, M., Mannix, J., Jackson, D. \& Hunt, G. E. (2012). Views and experiences of mental health nurses working with undergraduate assistants in nursing in an acute mental health setting. International Journal of Mental Health Nursing, 21, 184-190.

Cowan, D., Brunero, S., Lamont, S. \& Joyce, M. (2015a). Direct care activities for assistants in nursing in inpatient mental health settings in Australia : A modified Delphi study. Collegian, 22, 53-60.

Cowan, D., Frame, N., Brunero, S., Lamont, S. \& Joyce, M. (2015b). Assistants' in nursing perceptions of their social place within mental health-care settings. International Journal of Mental Health Nursing, 24, 439-447.

Duffield, C., Roche, M. A., Twigg, D., Williams, A., Rowbotham, S. \& Clarke, S. (2018). Adding unregulated nursing support workers to ward staffing: exploration of a natural experiment. Journal of Clinical Nursing, 27, 37683779.

Duffield, C., Roche, M. A., Wise, S. \& Debono, D. (2020). Harnessing ward level administrative data and expert knowledge to improve staffing decisions: a multi-method case study. Journal of Advanced Nursing, 76, 287296.

Duffield, C., Twigg, D., Roche, M. A., Williams, A. \& Wise, S. (2019). Uncovering the disconnect between nursing workforce policy intentions, implementation, and outcomes: lessons learned from the addition of a nursing assistant role. Policy, Politics, \& Nursing Practice, 20, 228-238.

Gabrielsson, S., Looi, G.-M. E., Zingmark, K. \& Sävenstedt, S. (2014). Knowledge of the patient as decision-making power: Staff members' perceptions of interprofessional collaboration in challenging situations in psychiatric inpatient care. Scandinavian Journal of Caring Sciences, 28, 784-792.

Gabrielsson, S., Sävenstedt, S. \& Olsson, M. (2016). Taking personal responsibility: Nurses' and assistant nurses' experiences of good nursing practice in psychiatric inpatient care. International Journal of Mental Health Nursing, 25, 434-443.

Gerace, A., Muir-Cochrane, E., O'Kane, D., Couzner, L., Palmer, C. \& Thornton, K. (2018). Assistants in nursing working with mental health consumers in the emergency department. International Journal of Mental Health Nursing, 27, 1729-1741.

Hasson, F., Keeney, S. \& McKenna, H. (2000). Research guidelines for the Delphi survey technique. Journal of Advanced Nursing, 32, 1008-1015. 
Kærnested, B. \& Bragadóttir, H. (2012). Delegation of registered nurses revisited: Attitudes towards delegation and preparedness to delegate effectively. Nordic Journal of Nursing Research, 32, 10-15.

Kaiser, J. A. \& Westers, J. B. (2018). Nursing teamwork in a health system: A multisite study. Journal of Nursing Management, 26, 555-562.

Kalisch, B. J. (2011). The impact of RN-UAP relationships on quality and safety. Nursing Management, 42, 16-22.

Kalisch, B. J., Lee, H. \& Rochman, M. (2010a). Nursing staff teamwork and job satisfaction. Journal of Nursing Management, 18, 938-947.

Kalisch, B. J., Lee, H. \& Salas, E. (2010b). The development and testing of the nursing teamwork survey. Nursing research, 59, 42-50.

Kalisch, B. J. \& Lee, K. H. (2013). Variations of nursing teamwork by hospital, patient unit, and staff characteristics. Applied Nursing Research, 26, 2-9.

Kizawa, Y., Tsuneto, S., Tamba, K., et al. (2012). Development of a nationwide consensus syllabus of palliative medicine for undergraduate medical education in Japan: a modified Delphi method. Palliative Medicine, 26, 744-752.

McComb, S. \& Simpson, V. (2014). The concept of shared mental models in healthcare collaboration. Journal of Advanced Nursing, 70, 1479-1488.

McNally, B. \& Blay, N. (2018). Assistants in Nursing: Scope of Practice and Pressure Injury Prevention. Australian Nursing and Midwifery Journal, 26, 46.

National Council of State Boards of Nursing (2016). National guidelines for nursing delegation. Journal of Nursing Regulation, 7, 5-14.

Needleman, J. (2017). Nursing skill mix and patient outcomes. BMJ Quality \& Safety, 26, 525-528.

NHS (2020). National Health Service Careers: Healthcare assistant. London: National Health Service.

NMBA (2015). Supervision guidelines for nursing and midwifery. Melbourne, VIC: Nurses and Midwifery Board of Australia.

NSW Health (2018). Employment of Assistants in Nursing (AIN) in NSW Health Acute Care. North Sydney: NSW Ministry of Health.

NSW Health (2019). Assistants in Nursing Working in the Acute Care Environment: Health Service Implementation Package. Revised Edition. In: Workforce Development and Innovation Branch: Health System Support Division (Ed). North Sydney: NSW Ministry of Health.

Paré, G., Cameron, A.-F., Poba-Nzaou, P. \& Templier, M. (2013). A systematic assessment of rigor in information systems ranking-type Delphi studies. Information \& Management, 50, 207-217.

Portelli, M., White, B., Wand, T., Haber, P. S. \& Glozier, N. (2018). 'Nurse specialling': Direct nursing observation in the emergency department compared to other wards of an urban teaching hospital in Sydney. Australasian Psychiatry, 26, 276-280.

Rahn, D. J. (2016). Transformational teamwork: Exploring the impact of nursing teamwork on nurse-sensitive quality indicators. Journal of Nursing Care Quality, 31, 262-268.

Ridoutt, L., Pilbeam, V. \& Perkins, D. (2014). Final report on workforce requirements in support of the 2014 National Review of Mental Health Programs and Services. National Mental Health Commission.

Rizzo, J. R., House, R. J. \& Lirtzman, S. I. (1970). Role conflict and ambiguity in complex organizations. Administrative science quarterly, 150-163.

Roche, M. A., Duffield, C., Friedman, S., Dimitrelis, S. \& Rowbotham, S. (2016). Regulated and Unregulated Nurses in the Acute Hospital Setting: Tasks Performed, Delayed or Not Completed. Journal of Clinical Nursing, 25, 153162.

Roche, M. A., Friedman, S., Duffield, C., Twigg, D. \& Cook, R. (2017). A comparison of nursing tasks undertaken by regulated nurses and nursing support workers: a work sampling study. Journal of Advanced Nursing, 73, 1421-1432. 
Ryan, L., Jackson, D., Woods, C. \& Usher, K. (2019). Intentional rounding - An integrative literature review. Journal of Advanced Nursing, 75, 1151-1161.

Salas, E., Sims, D. E. \& Shawn Burke, C. (2005). Is there A "big five" in teamwork? Small Group Research, 36, 555-599.

Scheffler, R. M., Cometto, G., Tulenko, K., Bruckner, T., Liu, J. X. \& Keuffel, E. L. (2016). Health workforce requirements for universal health coverage and the Sustainable Development Goals-Background paper N. 1 to the WHO Global Strategy on Human Resources for Health: Workforce 2030. Geneva, Switzerland: World Health Organization.

Schwartz, S. (2019). Educating the Nurse of the Future-Report of the Independent Review into Nursing Education. Commonwealth of Australia.

Twigg, D., Myers, H., Duffield, C., Pugh, J., Gelder, L. \& Roche, M. A. (2016). The impact of adding assistants in nursing to acute care hospital ward nurse staffing on adverse patient outcomes: An analysis of administrative health data. International Journal of Nursing Studies, 63, 189-200.

Vázquez-Ramos, R., Leahy, M. \& Hernández, N. E. (2007). The Delphi Method in Rehabilitation Counseling Research. Rehabilitation Counseling Bulletin, 50, 111-118.

Wagner, E. A. (2018). Improving Patient Care Outcomes Through Better Delegation-Communication Between Nurses and Assistive Personnel. Journal of Nursing Care Quality, 33, 187-193.

White, M. J., Gutierrez, A., Davis, K., Olson, R. \& McLaughlin, C. (2011). Delegation knowledge and practice among rehabilitation nurses. Rehabilitation nursing, 36, 16-24.

WHO (2016). Global strategy on human resources for health: workforce 2030.

\section{Acknowledgements}

We extend our thanks to all participants in both phases of this study. We also acknowledge Naomi Kayesa who contributed to Phase 2 data collection and the initial literature search.

\section{Funding}

This study received no specific funding.

\section{Conflicts of Interest}

None.

\section{Ethical statement}

The authors confirm that the Human Research Ethics Committee of Northern Sydney Local Health District considered Phase 1 of this study 'an activity not requiring HREC review', and that ethical approval was granted for Phase 2 of this study by the same committee (LNR/18/HAWKE/6, 8 May 2018).

\section{Author contributions}

MAR: Conceptualisation, Methodology (Phase 2), Data Curation, Formal Analysis, Writing - Original Draft, Supervision, Writing Review and Editing; SG: Conceptualisation, Methodology (Phase 2), Writing - Original Draft, Project Administration; XL:

Conceptualisation, Methodology (Phase 1), Data Curation, Formal Analysis, Project Administration; MJ: Conceptualisation, Writing Review and Editing, Project Administration; CR: Writing - Original Draft. 